\title{
Receptors, G proteins, and integration of calcium signalling
}

\author{
Thomas Gudermann ${ }^{1,2,3}$ • Michael Bader ${ }^{4,5,6,7}$
}

Published online: 21 August 2015

(C) Springer-Verlag Berlin Heidelberg 2015

All living cells in a multicellular organism critically depend on efficient ways of intercellular communication. One of the most extensively used transmembrane signalling module in nature is the $G$ protein-mediated signalling system $[1,2]$. $G$ protein-coupled receptors, G proteins, and effectors are expressed in all mammalian cells and upon activation evoke a host of cellular responses. Calcium is an ideal second messenger in cells, because due to the enormous concentration gradient of over 4 orders of magnitude many external stimuli can rapidly elevate the intracellular calcium concentration $\left(\left[\mathrm{Ca}^{2+}\right]_{\mathrm{i}}\right)$ and set in motion a variety of cellular processes like contraction, secretion, and gene transcription. Activation of phospholipase C (PLC)- $\beta$ isoforms by G protein-coupled receptors or of PLC- $\gamma$ by receptor tyrosine kinases results in the hydrolysis of phosphatidylinositol-4,5-bisphosphate $\left(\mathrm{PIP}_{2}\right)$ and the resultant formation of the second messengers diacylglycerol (DAG) and inositol 1,4,5-trisphosphate $\left(\mathrm{IP}_{3}\right)$ [3]. The ensuing rise of $\left[\mathrm{Ca}^{2+}\right]_{\mathrm{i}}$ is brought about by entry of external

Thomas Gudermann

Thomas.Gudermann@1rz.uni-muenchen.de

Michael Bader

mbader@mdc-berlin.de

1 Walther Straub Institute of Pharmacology and Toxicology, University of Munich, Munich, Germany

2 DZHK (German Centre for Cardiovascular Research), Munich Heart Alliance, Munich, Germany

3 Comprehensive Pneumology Center Munich (CPC-M), German Center for Lung Research, Munich, Germany

4 Max Delbrück Center for Molecular Medicine (MDC), Berlin-Buch, Germany

5 Charité-University Medicine, Berlin, Germany

6 Institute of Biology, University of Lübeck, Lübeck, Germany

7 Federal University of Minas Gerais, Belo Horizonte, Brazil
$\mathrm{Ca}^{2+}$ via nonselective cation channels in the plasma membrane, a process referred to as receptor-operated $\mathrm{Ca}^{2+}$ entry (ROCE) and $\mathrm{IP}_{3}$-induced release of $\mathrm{Ca}^{2+}$ from internal stores. The depletion of intracellular $\mathrm{Ca}^{2+}$ stores is the trigger for $\mathrm{Ca}^{2+}$ entry through highly $\mathrm{Ca}^{2+}$ selective plasma membrane ion channels. The latter phenomenon is called store-operated $\mathrm{Ca}^{2+}$ entry (SOCE) $[4,5]$.

In the recent past, novel biophysical, genetic, and pharmacological approaches have granted deeper insight into single components of such complex signalling cascades. However, the functional interplay of defined signalling modules still remains ill understood. As a springboard to disentangle the intricacies of cellular $\mathrm{Ca}^{2+}$ signalling sparked by receptors and $\mathrm{G}$ proteins, an international symposium was organized at the Max Delbrück Center in Berlin, Germany, November 21-22, 2014, supported by the German Center for Cardiovascular Research (DZHK), Max Delbrück Center, Berlin-Buch, and Transregional Collaborative Research Center 152 (TRR 152). The scientific aim of this symposium was to bring together the receptor/cyclic nucleotide and calcium signalling communities in order to better understand the integration of cellular signalling with a special focus on the cardiovascular system (Fig. 1). Receptors, $\mathrm{G}$ proteins, and $\mathrm{Ca}^{2+}$ permeable ion channels are central signalling modules orchestrating cardiovascular functions. The symposium had the focus on the integration of receptor, $G$ protein-, and calcium-mediated cellular signals underlying complex cellular functions. Because of his role as a catalyst of our advance in knowledge in this research area, the organizers also sought to feature the lifetime achievements of Lutz Birnbaumer as a major international player in $\mathrm{G}$ protein and $\mathrm{Ca}^{2+}$ signalling research for over five decades. A synoptic approach was chosen to attain a deeper mechanistic understanding of physiological regulation and pathologic dysregulation and to highlight novel pharmacological intervention strategies. 


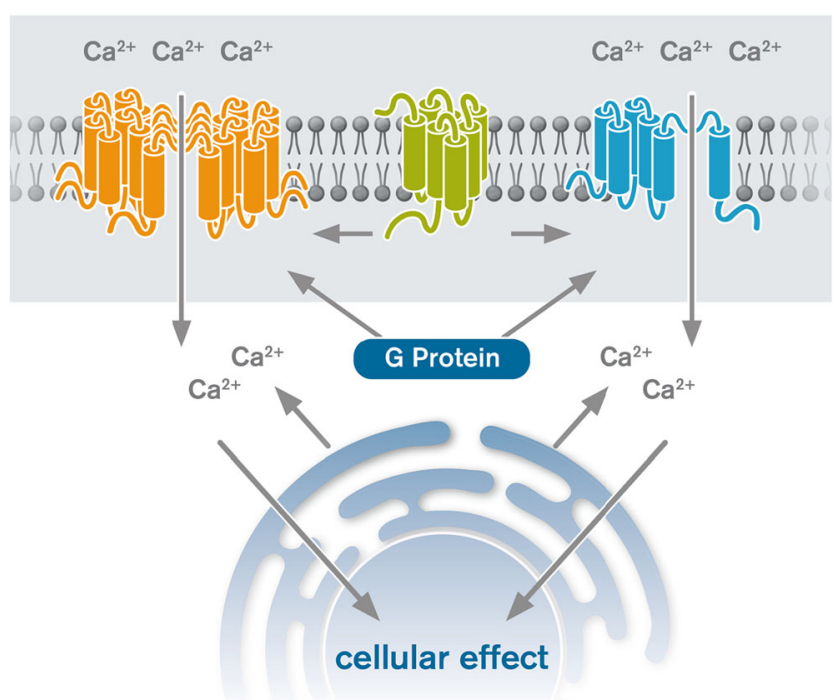

Fig. 1 Receptors, $\mathrm{G}$ proteins, and integration of cellular $\mathrm{Ca}^{2+}$ signalling. The activation of many $\mathrm{G}$ protein-coupled receptors (green) results in transient elevation of intracellular $\mathrm{Ca}^{2+}$ concentrations mediated by release of $\mathrm{Ca}^{2+}$ from intracellular organelles and influx through various cation channels (orange, blue). As a second messenger, $\mathrm{Ca}^{2+}$ initiates a vast array of cellular effects like contraction, secretion, and gene transcription. However, intracellular $\mathrm{Ca}^{2+}$ feeds back to modify the function of ion channels and other signalling proteins. Integrating the intricate relationships between receptors, $\mathrm{G}$ proteins, and cellular $\mathrm{Ca}^{2+}$ signalling to be able to predict cellular responses is a challenging task for future research

This issue of the Journal of Molecular Medicine has invited papers of eminent researchers in their respective fields who participated in the symposium to raise scientific awareness of therapeutic opportunities resulting from a more holistic understanding of cellular signalling pathways.

With a personal account of the development of cellular signal transduction over the last 50 years, Lutz Birnbaumer sets the stage for this review series [6]. In his article, Birnbaumer highlights pivotal discoveries in cellular signalling that turned out to be "game changers" revolutionizing our understanding of signal transduction. Starting from the activation mechanism of adenylyl cyclase by hormone receptors, he describes how $\mathrm{G}$ proteins were discovered and how eventually $G$ protein-coupled receptors entered the stage. A prime example for the relevance of active communication between different science communities is given by the discovery of G proteins: The parallelisms between transducin, the heteromeric GTPase in the retina activated by the seven-transmembrane protein rhodopsin, had been completely missed by the adenylyl cyclase aficionados for quite a number of years. The fact that scientific knowledge is often propelled forward by novel techniques granting unprecedented looks at details is illustrated by the long futile search for a postulated allosteric $\mathrm{Mg}^{2+}$ binding site in $\mathrm{G}$ proteins: It was not before the successful crystallization of G protein $\alpha$ subunits in the mid-1990s of the last century that researchers came to realize that the long sought allosteric site was the GTP-coordinating $\alpha$ subunit itself.

The light receptor rhodopsin was the first $G$ proteincoupled receptor whose overall structure and sequence was known, but only after the cloning of the various adrenergic receptors by Robert Lefkowitz [7] a new large protein family stepped into the limelight. The cloning and functional characterization of $\mathrm{G}_{\mathrm{q} / 11}$-coupled receptors stoked Lutz Birnbaumer's interest in $\mathrm{Ca}^{2+}$ signalling. He embarked on the cloning of members of the transient receptor potential (TRP) family of cation channels, termed after a Drosophila mutant with impaired vision, assuming that refilling of internal $\mathrm{Ca}^{2+}$ stores is mediated by TRPC proteins [8]. While this notion has remained a much contended issue over many years, there is compelling evidence by now that $\mathrm{Ca}^{2+}$ entry following store depletion, i.e., SOCE, is mainly mediated by the STIMOrai signalling module [9]. However, the question remained whether Orai requires TRPC proteins for proper function. Birnbaumer was a prominent proponent of the latter concept and answered the question himself: He genetically inactivated all seven TRPC homologues in mice and observed that the animals were alive, without gross abnormalities, and had perfectly preserved SOCE in fibroblasts. Thus, Orai channels operate in the absence of TRPC proteins that have been described to participate in a wide spectrum of physiological functions to maintain body homeostasis and were touted as promising therapeutic targets $[5,10,11]$. The seminal observation that a mouse devoid of all seven TRPC channels can survive is another "game changer" posing important questions to be answered in future studies.

As mentioned before, adrenoceptors are commonly regarded as archetypal $G$ protein-coupled receptors and adrenergic signalling controls many essential physiological systems. In his contribution, Martin Lohse [12] provides a concise historical account of the development of the field and then addresses issues at the forefront of current receptor research. Again, groundbreaking technological advances paved the way for unprecedented insight: The successful X-ray crystallography of G protein-coupled receptors, pioneered by Brian Kobilka [13], allowed a hitherto unknown detailed view on receptor and subsequently $G$ protein activation. However, several important questions remain: Spectroscopic studies with rhodopsin taught us that there is more than just one active receptor conformation. Most probably, this paradigm also applies to $G$ protein-coupled neurotransmitter and hormone receptors. Using modern spectroscopic approaches, researchers made the puzzling observation that receptor activation occurs with time constants of approximately $50 \mathrm{msec}$, roughly ten times faster than $G$ protein activation. The reason for this unexpected phenomenon still remains elusive. With regard to the production of second messengers such as cAMP, Lohse coins the notion of "signalling waves" 
elicited by membranous versus internalized receptors. Furthermore, the diffusion of second messengers in the cell is not free as elegantly shown by Martin Lohse in cardiac myocytes [14]: cAMP generated by $\beta_{2}$-adrenergic receptors in t-tubules cannot diffuse freely, but is confined and has local effects. This may have a pathophysiological bearing, because such cAMP microdomains can hardly be observed in the failing heart. The situation is even complicated further by the well-established phenomenon of agonist-dependent differential signalling, also called "biased" signalling. These and other examples illustrate that GPCR signalling may be organized in complex spatio-temporal patterns coding additional biological information that still needs to be deciphered.

Pathophysiological aspects are highlighted in the review article by Christian Weber [15] who focuses on the role of chemokines and their receptors in atherosclerosis. Over the years, atherosclerosis has transformed from a mainly metabolic to a chronic inflammatory disease orchestrated by a complex pathophysiology [16]. Chemokines have been recognized as key regulators in atherogenesis exerting their effects by binding to $G$ protein-coupled receptors. In his contribution, Weber focuses on two chemokines: macrophage migration inhibitory factor (MIF) and CXCL12. While MIF appears to play a pro-inflammatory, and thus pro-atherogenic role, CXCL12 may have a protective role. While the perspective of chemokine-based therapies are vividly discussed in the literature [17], the interactions between different chemokines and between different cell types involved in atherogenesis are far from being understood. In the future, conclusive answers to these open questions may offer specific, targeted chemokine-based therapeutic options.

The role of $G$ proteins in vascular smooth muscle cells is at the heart of Stefan Offermanns' manuscript [18]. In the past years, Offermanns made seminal contributions to our understanding of the physiological role of $\mathrm{G}_{\mathrm{q} / 11}$ and $\mathrm{G}_{12 / 13}$ proteins in vivo by creating a host of constitutive and conditional knock-out mouse models. Vascular smooth muscle cells harbor a certain plasticity, because under certain hemodynamic conditions or after vascular injury, they lose their contractile, differentiated phenotype and become dedifferentiated, synthetic, and more motile. Physiological vascular regulation such as the myogenic response $[19,20]$ as well as the balance between these two extreme ends of a differentiation spectrum is controlled by $\mathrm{G}$ protein-coupled receptors. In his recent work, Offermanns and other laboratories provided compelling evidence to show that pro-contractile $G$ protein signals are critical regulators of vascular smooth muscle differentiation as well. Vascular smooth muscle is a prominent cell model to illustrate how receptors, G proteins, cyclic nucleotides, and $\mathrm{Ca}^{2+}$ signals are inextricably intertwined to determine cell function and promote cell fate decisions. The more we understand about this complex interplay, the more likely we will be able to devise new pharmacological strategies to interfere with smooth muscle function and dysfunction in physiology and pathophysiology.

In a final review article, Jana Hartmann and Arthur Konnerth [21] summarize recent advances in our appreciation of synaptic transmission in central mammalian neurons. It goes without saying that neurons are yet another prominent example of highly differentiated cells whose proper functioning needs to be orchestrated by a precise integration of various signalling modules. As introduced before, TRPC channels are activated downstream of PLC-coupled receptors [22]. The expression of one of the TRPC family members, TRPC 3 , is highest in cerebellar Purkinje cells where TRPC 3 is a central component of glutamatergic metabotropic synaptic transmission. In TRPC3-deficient knock-out mice, a characteristic slow excitatory postsynaptic current (slow EPSC) is completely ablated resulting in characteristic motor coordination deficits. The activation mechanism of TRPC3 is still surrounded by quite some controversy, because the vast majority of functional data was acquired in heterologous overexpression systems. Hartmann and Konnerth characterized the functional characteristics of TRPC 3 in native neurons and concluded that in contrast to non-neuronal cells STIM1 impacts TRPC3 gating not by direct protein-protein interaction but rather by the regulation of $\left[\mathrm{Ca}^{2+}\right]_{\mathrm{i}}$ that has a permissive role for TRPC 3 activation in neurons.

Participating in cellular signalling conferences over the last 20 years, one could repeatedly hear that $G$ proteins had finally come of age and there would be hardly anything interesting to discover in the years to come. The compilation of review articles in this issue of the Journal of Molecular Medicine has proven these augurs wrong, since we have witnessed an explosion of knowledge over the last decades. One great challenge for the future lies in the successful integration of the bewildering complexity of signalling molecules and pathways in unifying models that are amenable to further experimental testing. As expected in science, new detailed molecular and physiological insight instantly provokes new exciting questions and opens up new translational avenues. Therefore, it will be interesting to see which kind of surprises cellular signalling still will come up with in the future.

\section{References}

1. Gilman AG (2012) Silver spoons and other personal reflections. Annu Rev Pharmacol Toxicol 52:1-19

2. Offermanns S (2003) G proteins as transducers in transmembrane signalling. Prog Biophys Mol Biol 83:101-130

3. Putney JW, Tomita T (2012) Phospholipase C signalling and calcium influx. Adv Biol Regul 52(1):152-164

4. Putney JW (2014) Origins of the concept of store-operated calcium entry. Front Biosci 3:980-984 
5. Abramowitz J, Birnbaumer L (2009) Physiology and pathophysiology of canonical transient receptor potential channels. FASEB J 23(2):297-328

6. Birnbaumer L (2015) From GTP and G proteins to TRPC channels: a personal account. J Mol Med. doi:10.1007/s00109-015-1328-5

7. Lefkowitz RJ (2004) Historical review: a brief history and personal retrospective of seven-transmembrane receptors. Trends Pharmacol Sci 25(6):413-422

8. Montell C (2011) The history of TRP channels, a commentary and reflection. Pflugers Arch - Eur J Physiol 461:499-506

9. Hogan PG, Rao A (2015) Store-operated calcium entry: mechanisms and modulation. Biochem Biophys Res Commun 460:40-49

10. Wu LJ, Sweet TB, Clapham DE (2010) International Union of Basic and Clinical Pharmacology.LXXVI. Current progress in the mammalian TRP ion channel family. Pharmacol Rev 62(3):381-404

11. Nilius B, Szallasi A (2014) Transient receptor potential channels as drug targets: from the science of basic research to the art of medicine. Pharmacol Rev 66(3):676-814

12. Lohse MJ (2015) The ins and outs of adrenergic signalling. J Mol Med. doi:10.1007/s00109-015-1323-x

13. Kobilka B (2013) The structural basis of G-protein-coupled receptor signalling (Nobel Lecture). Angew Chem Int Ed 52:6380-6388

14. Nikolaev VO, Moshkov A, Lyon AR, Miragoli M, Novak P, Paur H, Lohse MJ, Korchev YE, Harding SE, Gorelik J (2010) $\beta_{2}$-adrenergic receptor distribution in heart failure changes cAMP compartmentation. Science 327(5973):1653-1657
15. van der Vorst EPC, Döring Y, Weber C (2015) Chemokines and their receptors in atherosclerosis. J Mol Med. doi:10.1007/s00109015-1317-8

16. Weber C, Noels H (2011) Atherosclerosis: current pathogenesis and therapeutic options. Nat Med 17(11):1410-1422

17. Ghadge S, Muhlstedt S, Ozcelik C, Bader M (2011) SDF-1alpha as a therapeutic stem cell homing factor in myocardial infarction. Pharmacol Ther 129:97-108

18. Althoff TF, Offermanns S (2015) G-protein-mediated signalling in vascular smooth muscle cells - implications for vascular disease. J Mol Med. doi:10.1007/s00109-015-1305-Z

19. Mederos y Schnitzler M, Storch U, Meibers S, Nurwakagari P, Breit A, Essin K, Gollasch M, Gudermann T (2008) $\mathrm{G}_{\mathrm{q}}$-coupled receptors as mechanosensors mediating myogenic vasoconstriction. EMBO J 27:3092-3103

20. Schleifenbaum J, Kassmann M, Szijarto I, Hercule H, Tano JY, Weinert S, Heidenreich M, Pathan A, Anistan Y, Alenina N et al (2014) Stretch-activation of angiotensin II type 1a receptors contributes to the myogenic response of mouse mesenteric and renal arteries. Circ Res 115:263-272

21. Hartmann J, Konnerth A (2015) TRPC3-dependent synaptic transmission in central mammalian neurons. J Mol Med. doi:10.1007/ s00109-015-1298-7

22. Hofmann T, Obhukhov AG, Schaefer M, Harteneck C, Gudermann T, Schultz G (1999) Direct activation of human TRPC6 and TRPC3 channels by diacylglycerol. Nature 397(6716):259-263 